Preprints are preliminary reports that have not undergone peer review.

They should not be considered conclusive, used to inform clinical practice, or referenced by the media as validated information.

\title{
Identification of risk factors for the severity of coronavirus disease 2019: a retrospective study of 163 hospitalized patients
}

Ye Tu

Tongji Hospital of Tongji Medical College of Huazhong University of Science and Technology

Ping Yang

Tongji Hospital of Tongji Medical College of Huazhong University of Science and Technology

Jingjing Wang

Tongji Hospital of Tongji Medical College of Huazhong University of Science and Technology

Xuebi Tian

Tongji Hospital of Tongji Medical College of Huazhong University of Science and Technology

Kai Wang

Huazhong University of Science and Technology Tongji Medical College

Chaolong Wang

Huazhong University of Science and Technology Tongji Medical College

Ailin Luo

Tongji Hospital of Tongji Medical College of Huazhong University of Science and Technology

Feng Gao ( $\nabla$ fgao@tjh.tjmu.edu.cn )

Tongji Hospital of Tongji Medical College of Huazhong University of Science and Technology

\section{Research}

Keywords: Novel coronavirus pneumonia, Clinical features, Risk factors

Posted Date: April 27th, 2020

DOI: https://doi.org/10.21203/rs.3.rs-23867/v1

License: (1) This work is licensed under a Creative Commons Attribution 4.0 International License. Read Full License 


\section{Abstract}

Background: To compare clinical features between moderate and severe cases with COVID-19, and screen factors associated with disease severity.

Methods: Demographic and clinical data were compared between moderate and severe cases. Logistic regression was performed for prognostic factors.

Results: 163 patients (median age 65.0 (56.8-71.0) years, 78 (47.9\%) females) were enrolled, including 87 (53.4\%) severe and 76 (46.6\%) moderate cases. $79(90.8 \%)$ severe and 59 (77.6\%) moderate cases had comorbidities, with hypertension and diabetes commonly presented. The most common symptoms were fever. Severe cases had higher lactate dehydrogenase (LDH), inflammatory cytokines and lymphopenia, eosinopenia on admission, and lower eosinophil and higher neutrophil counts from admission to day 13 and 19. Multivariable regression showed that neutrophilia, eosinopenia, high LDH and D-dimer were associated with severe COVID-19. In receiver operating characteristic curve analysis, LDH, eosinophil and neutrophil + eosinophil + LDH + D-dimer combination, with area under curve of $0.86,0.76$ and 0.93 , predicted severe illness with high sensitivity $(82.8 \%, 83.3 \%, 88.0 \%)$ and specificity $(68.4 \%, 84.2 \%, 81.3 \%)$.

Conclusions: Eosinopenia, higher LDH and neutrophil + eosinophil + LDH + D-dimer combination on admission were powerful indicators of severe COVID-19. Dynamic changes of neutrophils and eosinophils may be used to evaluate disease progression.

\section{Introduction}

Coronavirus disease 2019 (COVID-19), a recent epidemic human disease, is caused by severe acute respiratory syndrome coronavirus-2 (SARS-CoV-2). The clinical spectrum of SARS-CoV-2 infection ranges from mild to critical illness. Initial report suggests that $81 \%$ patients with COVID-19 were mild, $14 \%$ were severe, and $5 \%$ were critically ill [1]. While mortality among infected patients ranged from $0.5-4 \%$ [2]. The median duration from symptom onset to intensive care unit (ICU) admission was 9 to 10 days, suggesting a gradual deterioration in more serious cases [2]. Thus, early identification of severe cases with COVID-19 and recognizing the initial signs of deterioration are crucial to determine how to best implement treatments.

In clinical practice, identification of severe COVID-19 is mainly based on the respiratory symptoms and radiographic findings. Besides the principal features of progressive respiratory failure in severe and critical illness, blood biochemical parameters represent the dynamic changes of inflammatory response and extra-pulmonary injury caused by SARS-CoV-2 infection. According to the Chinese management guideline for COVID-19, several laboratory findings, including leucocyte count, interleukin-6 (IL-6), C-reactive protein (CRP) and lactate, have been proposed to be clinical early-warning indicators of severe and critical cases [3]. However, their sensitivity and specificity for predicting disease severity have not been fully determined yet. With the further understanding of COVID-19, this guideline also needs more information to update. It is noteworthy that the abnormalities of blood cell counts, lactate dehydrogenase (LDH), coagulation profile, inflammatory mediators frequently occur in COVID-19 [4,5]. Several studies based on relatively small sample sizes reported that T lymphocytes, interferonY, platelet-to-lymphocyte ratio, CRP and procalcitonin were associated with disease severity of COVID-19 [5-7]. However, there is insufficient data about the differences between moderate and severe COVID-19. In this single-centered, retrospective, observational study, we comprehensively compared the clinical and laboratory features between moderate and severe cases, aiming to screen the risk factors associated with the disease severity of COVID-19.

\section{Methods}

\section{Study design and participants}

This retrospective study included adult inpatients ( $\geq 18$ years of age) with COVID-19 from Jan 28 to Feb 29, 2020, at Tongji Hospital, Tongji Medical College, Huazhong University of Science \& Technology, which is a designated hospital for severe and critical patients with COVID-19 in Wuhan, China. Patients diagnosed with COVID-19 according to the WHO interim guidance were screened, and those classified as moderate or severe illness of COVID-19 were enrolled in this study. Patients received corticosteroid treatments within 7 days were excluded. To determine the risk factors associated with COVID-19 severity more accurately, it is of great importance to made strict discrimination between severe and critical cases. Fatal patients were also excluded from this study.

The disease severity was defined according to the Chinese management guideline for COVID-19 (version 7.0) [3]. Moderate COVID-19 was designated for patients with fever, respiratory and other symptoms, and imaging features of pneumonia. Severe COVID-19 was designated when the patients met one of the following criteria: (a) Shortness of breath with respiratory frequency $\geq 30$ per minute; (b) Oxygen saturation $\leq 93 \%$ in the resting state; (c) Oxygenation index (artery blood oxygen partial pressure/inspired oxygen fraction, $\mathrm{PaO}_{2} / \mathrm{FiO}_{2}$ ) $\leq 300 \mathrm{mmHg}$. 
The study protocol was reviewed and approved by the Institutional Ethics Board of Tongji Hospital, Tongji Medical College, Huazhong University of Science \& Technology (TJ-IRB20200361). Written informed consent was waived owing to the rapid emergence of this infectious disease.

\section{Data Collection}

Data including demographic information, comorbidities, symptoms, signs, chest computed tomographic (CT) scans, laboratory findings, and treatments were extracted from electronic medical records. All data were checked and reviewed by a trained team of physicians.

Laboratory findings on admission and during hospitalization were collected, including blood route, blood biochemical examinations ( aminotransferase, bilirubin, albumin, lactate dehydrogenase [LDH], creatinine, blood glucose, etc.), myocardial enzymes, coagulation profile, cytokine profile, N-terminal pro-B-type natriuretic peptide (NT-proBNP), high sensitivity C-reactive protein (hsCRP), procalcitonin, and ferritin.

Throat swab samples were collected and used for SARS-CoV-2 detection using real-time RT-PCR. A total of $200 \mu \mathrm{L}$ sample was used for laboratory confirmation of COVID-19 with the respiratory sample RNA isolation kit (Yuesui equipment No. 20170583 and 20150302, Da'an gene Co., Ltd, Sun Yat-sen University, China) according to the manufacturer's instructions. A cycle threshold value less than 40 was defined as positive response.

\section{Statistical analysis}

Continuous and categorical variables were presented as median (IQR) and $n(\%)$, respectively. We used the Mann-Whitney U test, $\chi^{2}$ test, or Fisher's exact test to compare differences between moderate and severe cases where appropriate. To explore the risk factors associated with disease severity, univariable and multivariable logistic regression models were utilized. The selection of candidate variables for univariable analysis was based on all measured variables which had been known or suspected to be associated with disease severity of COVID-19. Multiple imputation was performed for missing data. We excluded variables from univariable analysis if their between-group differences were not significant, if number of event was too small to calculate odds ratio, and if missing data of variable was more than $15 \%$. If highly correlated, the variable with lower $p$ value was entered as independent variable. All variables with $P<0.05$ in univariable analysis were entered into multivariate analysis. Discrimination of the models was quantified by using the area under a receiver operating characteristic (AUC-ROC) curve, the model fit was determined with Nagelkerke's $\mathrm{R}^{2}$ index, and the goodness of fit of model was assessed by HosmerLemeshow test. The optimal cut-off value for discrimination of disease severity was chosen using Younden's index. All statistical analyses were conducted using SPSS (Statistical Package for the Social Sciences) version 19.0 software (SPSS Inc.) with the level of significance set to $\mathrm{a}=0.05$ (two-tailed).

\section{Results}

\section{Demographics and baseline characteristics of moderate and severe patients}

191 patients with COVID-19 were enrolled in this study. After excluding 24 patients who were not confirmed by SARS-CoV-2 RNA detection as of Feb 29, 2020, and 4 patients with incomplete core information in their electronic medical records, we included 163 patients in the final analysis. 87 (53.4\%) of the 163 patients were clinically diagnosed as severe COVID-19, whereas 76 (46.6\%) as moderate. The median age of the 163 patients was 65.0 years (range, 27-89 years), and nearly half were male. There were more male patients in severe cases, while opposite in moderate cases. Most patients in two groups had comorbidities, which was more pronounced in severe cases, with hypertension and diabetes commonly presented (Table 1). And severe patients were more likely to have hypertension. Among the 163 patients, the most common symptom was fever, followed by dry cough, expectoration, fatigue, dyspnea, diarrhea, and myalgia. Compared with moderate cases, less severe cases experienced expectoration. Only severe cases experienced dyspnea, with higher systolic blood pressure and increased respiratory rate on admission. The median time from illness onset to admission and hospital stay were similar between moderate and severe cases (13 vs $12 ; P>0.05 ; 34$ vs $36 ; P>0.05$; respectively).

\section{Laboratory and radiographic findings}

We observed extensive differences in laboratory findings between two groups (Table 2). 22 severe patients and only 3 moderate patients had leukocytosis (white blood cell [WBC] count $\geq 10 \times 10^{9} / \mathrm{L}$ ) on admission. Increased neutrophils $\left(\geq 6.3 \times 10^{9} / \mathrm{L}\right.$ ) were more frequent in severe patients $(46.0 \%$ vs $6.6 \% ; P<0.001)$. More severe patients had lymphopenia (lymphocyte count $<0.8 \times 10^{9} / \mathrm{L}$ ) and eosinopenia (eosinophil 
count $<0.02 \times 10^{9} / \mathrm{L}$ ) than moderate patients. Severe patients had higher WBC and neutrophil counts, as well as lower lymphocyte and eosinophil counts.

Levels of aspartate aminotransferase (AST), total bilirubin, direct bilirubin, indirect bilirubin, creatinine, glucose, high-sensitivity cardiac troponin I (hs-cTnl), creatine kinase isoenzyme-MB (CK-MB), procalcitonin, D-dimer, prothrombin time (PT) and international normalized ratio (INR) were higher in severe patients than moderate patients. Serum albumin was significantly lower in severe patients than moderate patients, and hypoalbuminemia (albumin < $32 \mathrm{~g} / \mathrm{L}$ ) was more frequent in severe patients. In addition, levels of LDH, NT-proBNP, hsCRP and ferritin were remarkably higher in severe patients. Of patients with available data, a bunch of inflammatory cytokines were remarkably higher in severe patients than moderate patients, including interleukin-2 receptor (IL-2R), IL-6, IL-8, IL-10 and tumor necrosis factor-a (TNF-a). And increased IL-2R, IL-6 and TNF-a were detected in majority of severe patients.

On admission, abnormalities in thoracic computed tomography (CT) scans were observed in all patients. The typical CT features in severe patients included multifocal ground-glass opacities and subpleural consolidation, rapidly evolved to mass shadows of high density in bilateral lungs. Whereas representative CT features in moderate patients were unilateral or bilateral ground-glass opacification.

\section{Temporal changes of blood cell counts during hospitalization}

The dynamic profile of blood route was tracked after admission, from day 4 to day 25 at 3-day intervals (Fig. 1 and Supplementary Table 1). WBC and neutrophil counts were constantly higher in severe patients than moderate patients from admission to day 16 and 19 , respectively, and decreased with recovery. In severe patients, WBC and neutrophil counts were highest on day 10 after admission. Eosinophil and lymphocyte counts were significantly lower in severe patients than moderate patients until day 13 and 19, respectively. In severe patients, lymphocyte and eosinophil counts were lowest on admission, gradually increased during hospitalization. Platelet counts were slightly lower in severe patients than moderate patients in the first week of hospitalization.

\section{Treatments, complications, and clinical outcomes}

Antiviral coverage (oseltamivir, arbidol or lopinavir/ritonavir) was similar between two groups. 85 severe patients and 57 moderate patients received empirical antibiotics (moxifloxacin or cephalosporin, etc). More severe patients were prescribed corticosteroids, immunoglobulin and thymosin. A large proportion of severe patients required high flow nasal cannula or non-invasive mechanical ventilation (Table 3). The most common complication in severe patients was respiratory failure (77.0\%). Less frequent complications in severe patients included acute cardiac injury (17.2\%), acute kidney injury (12.5\%) and shock (9.2\%), most of which were not observed in any moderate cases. As of March 15, 2020, 133 (81.6\%) patients had fully recovered and been discharged, 30 (27.4\%) were still in treatment.

\section{Exploring independent factors for discrimination of disease severity}

Univariate analysis identified 10 variables associated with disease severity, including hypertension, sex (male), neutrophil count, eosinophil count $\left(<0.04 \times 10^{9} / \mathrm{L}\right)$, lymphocyte count, LDH, albumin, creatinine, PT and D-dimer. A multiple logistic regression model was fitted using these variables. Backward elimination at this point removed 6 variables from the model. The final independent variables in the model were neutrophil count, eosinophil count $\left(<0.04 \times 10^{9} / \mathrm{L}\right)$, LDH and D-dimer with odds ratios of $1.34,19.93,1.02$ and 1.15 , respectively (Table 4$)$.

Among 4 variables, LDH demonstrated the highest predictive value to discriminate COVID-19 severity with an AUC of 0.86 and a cut-off level of 310 in ROC curve analysis. Moreover, we used several eosinophil count cutoff values to discriminate severe and moderate cases. The result showed that the best eosinophil cell count cut-off value was $0.04 \times 10^{9} / \mathrm{L}$, with AUC of 0.82 , (95\% Cl, $\left.0.77-0.88\right)$, and sensitivity of $83.3 \%$ and specificity of $68.4 \%$ (Table 5 , Figure 2). Nagelkerke pseudo $\mathrm{R}^{2}$ for the model was 0.695 , indicating that 4 variables accounted for $69.5 \%$ of the variance in COVID-19 severity. The Hosmer-Lemeshow test indicated satisfactory fit $(P=0.934)$. The performance of combined variables in modeling for discriminating disease severity was tested. Neutrophil + eosinophil $\left(<0.04 \times 10^{9} / \mathrm{L}\right)+\mathrm{LDH}+\mathrm{D}-\mathrm{dimer}$ combinations demonstrated high predictive values (Table 5 and Fig. 2).

\section{Discussion}

This study identified several risk factors for the severity of COVID-19. In particular, neutrophilia, eosinopenia, higher LDH and D-dimer on admission were associated with the severity of COVID-19. LDH, eosinophil $\left(<0.04 \times 10^{9} / \mathrm{L}\right)$ and neutrophil + eosinophil + LDH + D-dimer combination were the independent indicators of the severe COVID-19 with relatively high sensitivity and specificity. Compared with

Page $4 / 14$ 
moderated cases, WBC and neutrophil counts were significantly higher in severe cases, while eosinophil and lymphocyte counts were markedly lower after admission, and then improved during hospitalization.

While previous studies reported a male predominance in SARS-CoV-2 infection [2, 5], this study further showed that male patients, particularly those with underlying comorbidities, were more liable to develop severe COVID-19. Although patients with chronic lung diseases were likely to be less susceptible to SARS-CoV-2 infection [8], we found no significant difference in proportion of chronic obstructive pulmonary disease between moderate and severe cases, likely due to the small samples of this study. Consistent with previous study [9], duration from illness onset to admission and hospital stay in moderate and severe cases had no difference. The most common symptom was fever, while other symptoms were different between two groups. Dyspnea tended to be more frequent in severe cases, while expectoration in moderate cases. On admission, severe cases presented higher blood pressure and increased respiratory rate.

leukocytosis and neutrophilia were more common in severe patients than moderate patients. Together with higher procalcitonin in severe patients, these findings suggested that secondary bacterial infection might develop in many severe cases. More frequent and persistent lymphopenia observed in severe patients indicated the weak immune function associated with serious illness. While increases in blood glucose, LDH, hs-cTnl, CK-MB, NT-proBNP, hsCRP, IL-2R, IL-6 and TNF-a were much more frequent and significant in severe patients than moderate patients, which were similar to previous studies [10]. Even the pathological study of COVID-19 showed that myocardium and endocardium were not significantly affected by SARS-CoV-2 infection [11], physicians should be alert to the possibile myocardial injury in severe cases with cardiovascular comorbidities.

Eosinophil counts in a larger proportion of severe patients were extremely low, even zero, which could also be observed in critical patients [12]. While eosinophil in most moderate patients were within normal range, similar to other studies [8]. We further confirmed that eosinopenia was the most significant risk factor for severe COVID-19 (OR = 19.86). Both infectious and noninfectious stimuli of acute inflammation could inhibit eosinophilia [13], suggesting that eosinopenia in severe COVID-19 may resulted from pneumonia or systemic inflammation. During virus infection, virus-neutralizing antibodies complex can initiates secondary inflammatory response through complement system, which may contribute to peripheral sequestration of eosinophils by localization in inflammatory region and eventually lead to lung injury [10].

Consistent with previous study [14], we found that SARS-CoV-2 infection could cause neutrophilia in severe patients, similar to the results in SARS [15]. Furthermore, after excluding the cases who received corticosteroids before admission, we confirmed that neutrophilia was associated with severe COVID-19. Cytokine storm and viral evasion of immune response contribute to disease severity. Neutrophils are the main source of chemokines and cytokines. Previously, neutrophilia was found in peripheral blood and lungs in SARS [16]. As a host immune response to SARS-CoV-2 infection, activated neutrophils may contribute to more serious cytokine storm and acute respiratory distress syndrome (ARDS) in severe COVID-19 [1].

So far there is still lack of dynamic profile of laboratory findings in COVID-19. This study found the decreased eosinophils and increased neutrophils in severe patients compared with moderate patients from admission to day 13 or 19 , respectively, when patients were clinically improved. While eosinophil and neutrophil counts in most patients with moderate COVID-19 remained within the normal ranges during hospitalization. This reasonably raises the possibility that temporal changes of eosinophil and neutrophil counts may reflect the pathophysiological course of COVID-19, such as disease deterioration, improvement, and therapeutic response. In this study, $74.7 \%$ of severe patients received corticosteroid treatment, which may affect the numbers of peripheral eosinophil and neutrophils, so the prognostic values of eosinophil and neutrophils should be interpreted with caution.

Increased LDH was detected in most COVID-19 cases, especially in nearly all of the severe patients, consistent with other studies [17], as well as the results in SARS [18]. We further found that LDH was the most valuable predictor for severe COVID-19. LDH has been reported to be an independent risk factor for severe/critical COVID-19 in patients with gastrointestinal symptoms [19].These observations seem to be similar to SARS, where elevated LDH and neutrophilia predicted poor outcomes [18]. As a non-specific enzyme, LDH is associated with the degree of tissue damage and severity of pneumonia. There was a significantly positive correlation between LDH and inflammation severity or extensive lung injury in COVID-19 with the initial CT features,[19] which were frequently observed in viral pneumonia [20]. Inverse correlation between PCR negative conversion rate and LDH concentration indicated viral clearance and predicted therapeutic response to SARS-CoV-2 infection [21].

Consistent with other studies [22, 23], the differences in abnormalities of coagulation function between moderate and severe patients with COVID-19 included increased PT, INR and D-dimer. The pathogenesis of COVID-19 has not been completely understood. Besides cytokine storm, coagulation dysfunction also account for disease severity, especially the balance between coagulation and fibrinolysis regulating diffuse alveolar damage and acute lung injury, as reported in SARS model [24]. SARS-CoV-2 likely shares similar inflammatory responses and lung injury with SARS-CoV. Previous study of COVID-19 revealed that the difference in D-dimer levels between deceased patients and 
survivors was higher than that between ARDS and non-ARDS patients [4], suggesting that varying degrees of disseminated intravascular coagulation might occur in severe and critical COVID-19.

This study has several limitations. First, only cases with typical moderate or severe COVID-19 were included to strictly discriminate the disease severity, which might results in potential selection bias. Second, this study was conducted at a single-center hospital with a small sample size, and the identified risk factors should be further validated. Third, it is noteworthy that the risk factors evaluated in this study were in cases who fully recovered from COVID-19, thus whether they contribute to recovery or disease deterioration remains unclear.

\section{Conclusion}

We provide the evidence that neutrophilia, eosinopenia, higher LDH and D-dimer were associated with the severity of COVID-19. And LDH, eosinopenia and neutrophil + eosinophil + LDH + D-dimer combination were powerful indicators of severe COVID-19 at an early stage. Dynamic changes of neutrophils and eosinophils during hospitalization may be helpful to evaluate the improvement of disease.

\section{Abbreviations}

COVID-19: Coronavirus disease 2019; SARS-CoV-2: Severe acute respiratory syndrome coronavirus 2; ICU: intensive care unit; WBC: white blood cell; ROC: Receiver operation characteristic; OR: Odds ratio; NT-proBNP: N-terminal pro-B-type natriuretic peptide; Hs-cTnl: highsensitivity cardiac troponin I; CK-MB: creatine kinase isoenzyme-MB; LDH: lactate dehydrogenase; IL-6: interleukin-6; CRP: C-reactive protein; PT: Prothrombin time; INR: International normalized ratio

\section{Declarations}

\section{Acknowledgments}

We acknowledge all health-care workers involved in the diagnosis and treatment of patients in Wuhan, China.

\section{Author contributors}

Y. T., P. Y., W., K. W., and F. G. collected, analyzed, and interpreted the data. Y. T., A. L., X. T., C. W., and F. G. conceived the study, delineated the hypothesis, and designed the study. Y. T., P. Y., and F. G. wrote the manuscript.

\section{Funding}

This work was funded by grants from the National Natural Science Foundation of China (Grant No. 81771199, 81601481).

\section{Availability of data and materials}

The raw data required to reproduce these findings cannot be shared at this time as the data also forms part of an ongoing study.

\section{Ethics approval and consent to participate}

The study was approved by the Institutional Ethics Board of Tongji Hospital, Tongji Medical College, Huazhong University of Science \& Technology (TJ-IRB20200361). Written informed consent was waived owing to the rapid emergence of this infectious disease.

\section{Consent for publication}

Not applicable.

\section{Competing interests}

The authors declare that they have no competing interests

\section{References}

1. Wu Z, McGoogan JM: Characteristics of and Important Lessons From the Coronavirus Disease 2019 (COVID-19) Outbreak in China: Summary of a Report of 72314 Cases From the Chinese Center for Disease Control and Prevention. JAMA 2020.

2. Yang X, Yu Y, Xu J, Shu H, Xia J, Liu H, Wu Y, Zhang L, Yu Z, Fang M, et al: Clinical course and outcomes of critically ill patients with SARS-CoV-2 pneumonia in Wuhan, China: a single-centered, retrospective, observational study. Lancet Respir Med 2020. 
3. National Health Commission of the People's Republic of China. Chinese management guideline for COVID-19 (version 7.0). March 3, 2020. http://www.nhc.gov.cn/yzygj/s7653p/202003/46c9294a7dfe4cef80dc7f5912eb1989/files/ce3e6945832a438eaae415350a8ce964.pdf.

4. Wu C, Chen X, Cai Y, Xia J, Zhou X, Xu S, Huang H, Zhang L, Zhou X, Du C, et al: Risk Factors Associated With Acute Respiratory Distress Syndrome and Death in Patients With Coronavirus Disease 2019 Pneumonia in Wuhan, China. JAMA Intern Med 2020.

5. Chen G, Wu D, Guo W, Cao Y, Huang D, Wang H, Wang T, Zhang X, Chen H, Yu H, et al: Clinical and immunologic features in severe and moderate Coronavirus Disease 2019. J Clin Invest 2020.

6. Qu R, Ling Y, Zhang YHZ, Wei LY, Chen X, Li XM, Liu XY, Liu HM, Guo Z, Ren H, Wang Q: Platelet-to-lymphocyte ratio is associated with prognosis in patients with coronavirus disease-19. Journal of Medical Virology 2020.

7. Li K, Wu J, Wu F, Guo D, Chen L, Fang Z, Li C: The Clinical and Chest CT Features Associated with Severe and Critical COVID-19 Pneumonia. Invest Radiol 2020.

8. Liu F, Xu A, Zhang Y, Xuan W, Yan T, Pan K, Yu W, Zhang J: Patients of COVID-19 may benefit from sustained lopinavir-combined regimen and the increase of eosinophil may predict the outcome of COVID-19 progression. Int J Infect Dis 2020.

9. Zhang JJ, Dong X, Cao YY, Yuan YD, Yang YB, Yan YQ, Akdis CA, Gao YD: Clinical characteristics of 140 patients infected with SARSCoV-2 in Wuhan, China. Allergy 2020.

10. Fu Y, Cheng Y, Wu Y: Understanding SARS-CoV-2-Mediated Inflammatory Responses: From Mechanisms to Potential Therapeutic Tools. Virol Sin 2020.

11. Xu Z, Shi L, Wang Y, Zhang J, Huang L, Zhang C, Liu S, Zhao P, Liu H, Zhu L, et al: Pathological findings of CoVID-19 associated with acute respiratory distress syndrome. Lancet Respir Med 2020, 8:420-422.

12. Du Y, Tu L, Zhu P, Mu M, Wang R, Yang P, Wang X, Hu C, Ping R, Hu P, et al: Clinical Features of 85 Fatal Cases of COVID-19 from Wuhan: A Retrospective Observational Study. Am J Respir Crit Care Med 2020.

13. Bass DA: Behavior of eosinophil leukocytes in acute inflammation. II. Eosinophil dynamics during acute inflammation. $J$ Clin Invest 1975, 56:870-879.

14. Qin C, Zhou L, Hu Z, Zhang S, Yang S, Tao Y, Xie C, Ma K, Shang K, Wang W, Tian DS: Dysregulation of immune response in patients with COVID-19 in Wuhan, China. Clin Infect Dis 2020.

15. Xiong Y, Sun D, Liu Y, Fan Y, Zhao L, Li X, Zhu W: Clinical and High-Resolution CT Features of the COVID-19 Infection: Comparison of the Initial and Follow-up Changes. Invest Radiol 2020.

16. Nicholls JM, Poon LL, Lee KC, Ng WF, Lai ST, Leung CY, Chu CM, Hui PK, Mak KL, Lim W, et al: Lung pathology of fatal severe acute respiratory syndrome. Lancet 2003, 361:1773-1778.

17. Wang D, Hu B, Hu C, Zhu F, Liu X, Zhang J, Wang B, Xiang H, Cheng Z, Xiong Y, et al: Clinical Characteristics of 138 Hospitalized Patients With 2019 Novel Coronavirus-Infected Pneumonia in Wuhan, China. JAMA 2020.

18. Tsui PT, Kwok ML, Yuen H, Lai ST: Severe acute respiratory syndrome: clinical outcome and prognostic correlates. Emerg Infect Dis 2003, 9:1064-1069.

19. Jin X, Lian JS, Hu JH, Gao J, Zheng L, Zhang YM, Hao SR, Jia HY, Cai H, Zhang XL, et al: Epidemiological, clinical and virological characteristics of 74 cases of coronavirus-infected disease 2019 (COVID-19) with gastrointestinal symptoms. Gut 2020.

20. Tsang OT, Chau TN, Choi KW, Tso EY, Lim W, Chiu MC, Tong WL, Lee PO, Lam BH, Ng TK, et al: Coronavirus-positive nasopharyngeal aspirate as predictor for severe acute respiratory syndrome mortality. Emerg Infect Dis 2003, 9:1381-1387.

21. Yuan J, Zou R, Zeng L, Kou S, Lan J, Li X, Liang Y, Ding X, Tan G, Tang S, et al: The correlation between viral clearance and biochemical outcomes of 94 COVID-19 infected discharged patients. Inflamm Res 2020.

22. Tang N, Li D, Wang X, Sun Z: Abnormal coagulation parameters are associated with poor prognosis in patients with novel coronavirus pneumonia. J Thromb Haemost 2020, 18:844-847.

23. Han H, Yang L, Liu R, Liu F, Wu KL, Li J, Liu XH, Zhu CL: Prominent changes in blood coagulation of patients with SARS-CoV-2 infection. Clin Chem Lab Med 2020.

24. Gralinski LE, Bankhead A, 3rd, Jeng S, Menachery VD, Proll S, Belisle SE, Matzke M, Webb-Robertson BJ, Luna ML, Shukla AK, et al: Mechanisms of severe acute respiratory syndrome coronavirus-induced acute lung injury. mBio 2013, 4.

\section{Tables}


Table 1 Demographics and baseline characteristics of patients with COVID-19

\begin{tabular}{|c|c|c|c|c|}
\hline Characteristics & All patients $(n=163)$ & Moderate cases $(n=76)$ & Severe cases $(n=87)$ & $P$ \\
\hline Age, years & $65.0(56.8-71.0)$ & $64.5(54.3-70.8)$ & $65.5(57.0-71.0)$ & 0.238 \\
\hline \multicolumn{5}{|l|}{ Sex } \\
\hline Males & $85 / 163(52.1 \%)$ & $33 / 76(43.4 \%)$ & $52 / 87$ (59.8\%) & 0.037 \\
\hline Femal & $78 / 163(47.9 \%)$ & $43 / 76(56.6 \%)$ & $35 / 87(40.2 \%)$ & .. \\
\hline Comorbidities & $138 / 163(84.7 \%)$ & $59 / 76(77.6 \%)$ & $79 / 87$ (90.8\%) & 0.020 \\
\hline Hypertension & $70 / 163(42.9 \%)$ & $24 / 76(31.6 \%)$ & $46 / 87$ (52.9\%) & 0.006 \\
\hline Diabetes & $41 / 163(25.2 \%)$ & $15 / 76(19.7 \%)$ & $26 / 87$ (29.9\%) & 0.136 \\
\hline Coronary heart disease & $24 / 163(14.7 \%)$ & $9 / 76(11.8 \%)$ & $14 / 87(16.1 \%)$ & 0.437 \\
\hline Cerebrovascular disease & $4 / 163(2.5 \%)$ & $2 / 76(2.6 \%)$ & $2 / 87(2.3 \%)$ & 1.000 \\
\hline Chronic obstructive pulmonary disease & $4 / 163(2.5 \%)$ & $0 / 76(0.0 \%)$ & $4 / 87(4.6 \%)$ & 0.124 \\
\hline Carcinoma & $7 / 163(4.3 \%)$ & $1 / 76(1.3 \%)$ & $6 / 87(6.9 \%)$ & 0.123 \\
\hline \multicolumn{5}{|l|}{ Signs and symptoms } \\
\hline Fever & $142 / 163$ (87.1\%) & $66 / 76(86.8 \%)$ & $76 / 87$ (87.4\%) & 0.922 \\
\hline Highest temperature, ${ }^{\circ} \mathrm{C}$ & $38.5(37.5-39.0)$ & $38.6(37.8-39.0)$ & $38.5(37.5-39.0)$ & 0.300 \\
\hline $38.1-39.0,{ }^{\circ} \mathrm{C}$ & $47 / 137$ (34.3\%) & $19 / 62(30.6 \%)$ & $28 / 75$ (37.3\%) & 0.641 \\
\hline$>39.0,{ }^{\circ} \mathrm{C}$ & $52 / 137$ (38.0\%) & $23 / 67$ (34.3\%) & 29/75 (38.7\%) & .. \\
\hline Dry cough & $69 / 163(42.3 \%)$ & $24 / 76$ (31.6\%) & 45/87 (51.7\%) & 0.059 \\
\hline Expectoration & $66 / 163(40.5 \%)$ & $40 / 76(52.6 \%)$ & 24/87 (27.6\%) & 0.001 \\
\hline Chest tightness & $32 / 163$ (19.6\%) & $12 / 76(15.8 \%)$ & $20 / 87$ (23.0\%) & 0.248 \\
\hline Dyspnea & $45 / 163(27.6 \%)$ & $0 / 76(0.0 \%)$ & 45/87 (51.7\%) & $<0.001$ \\
\hline Fatigue & $51 / 163(31.3 \%)$ & $23 / 76(30.3 \%)$ & $28 / 87(32.2)$ & 0.792 \\
\hline Diarrhea & $47 / 163(28.8 \%)$ & $18 / 76(36.8 \%)$ & $29 / 87$ (21.8\%) & 0.175 \\
\hline Myalgia & $28 / 163(17.2 \%)$ & $17 / 76(22.4 \%)$ & $11 / 87$ (12.6\%) & 0.101 \\
\hline Nausea/vomiting & $7 / 163(4.3 \%)$ & $3 / 76(3.9 \%)$ & $4 / 87(4.6 \%)$ & 1.000 \\
\hline Headache & $18 / 163(11.0 \%)$ & $6 / 76(17.1 \%)$ & $12 / 87$ (14.9\%) & 0.231 \\
\hline Systolic pressure, $\mathrm{mmHg}$ & $132.0(120.0-142.3)$ & $130.0(120.0-138.8)$ & $136.0(120.0-146.0)$ & 0.121 \\
\hline$>140 \mathrm{mmHg}$ & $46 / 154$ (29.9\%) & $13 / 69(18.8 \%)$ & $33 / 86$ (38.4\%) & 0.008 \\
\hline Diastolic pressure, $\mathrm{mmHg}$ & $80.0(74.0-89.3)$ & $80.0(73.5-89.0)$ & $80.0(74.0-90.0)$ & 0.850 \\
\hline$>90 \mathrm{mmHg}$ & $32 / 154(20.8 \%)$ & $12 / 69(17.4 \%)$ & $20 / 86(23.3 \%)$ & 0.248 \\
\hline Mean arterial pressure, $\mathrm{mmHg}$ & $97.3(91.3-105.3)$ & $96.7(90.3-102.6)$ & $99.7(91.6-107.4)$ & 0.369 \\
\hline Heart rate, beats per min & $90.0(83.5-104.0)$ & $90.0(80.0-102.8)$ & $92.0(85.0-104.5)$ & 0.304 \\
\hline Respiratory rate, breaths per min & $20.0(20.0-23.0)$ & $20.0(20.0-22.0)$ & $22.0(20.0-24.0)$ & $<0.001$ \\
\hline$\geq 30$ & $12 / 158(7.6 \%)$ & $0 / 71(0.0 \%)$ & $12 / 87$ (13.8\%) & 0.001 \\
\hline Duration from illness onset to admission, days & $12.0(8.0-17.0)$ & $13.0(8.0-19.0)$ & $12.0(8.0-16.0)$ & 0.176 \\
\hline Duration from admission to discharge, days & $35.0(27.0-41.0)$ & $34.0(27.0-40.0)$ & $36.0(27.0-43.0)$ & 0.233 \\
\hline Duration from illness onset to discharge, days & $48.0(40.0-54.5)$ & $48.0(40.0-54.0)$ & $48.5(39.0-55.0)$ & 0.743 \\
\hline
\end{tabular}

Abbreviations: COVID-19 coronavirus disease 2019. Data were median (IQR) or n/N (\%), where $\mathrm{N}$ was the total number of patients with available data. P 
values comparing moderate and severe cases were from $\chi \square$ test, Fisher's exact test, or Mann-Whitney U test. $P<0.05$ was considered stratistically significant. 
Table 2 Laboratory findings of patients with COVID-19

\begin{tabular}{|c|c|c|c|c|}
\hline Laboratory findings & All patients $(n=163)$ & Moderate cases $(n=76)$ & Severe cases $(n=87)$ & $P$ \\
\hline White blood cell count, $\times 10 \square / \mathrm{L}$ & $6.2(4.7-8.5)$ & $5.6(4.5-6.9)$ & $7.0(5.0-10.0)$ & 0.001 \\
\hline$<4$ & $19 / 163(11.7 \%)$ & $10 / 76(13.2 \%)$ & $9 / 87(10.3 \%)$ & 0.577 \\
\hline $4-10$ & $119 / 163(73.0 \%)$ & $63 / 76(82.9 \%)$ & $56 / 87(64.4 \%)$ &.. \\
\hline$\geq 10$ & $25 / 163(15.3 \%)$ & $3 / 76(3.9 \%)$ & $22 / 87(25.3 \%)$ & .. \\
\hline Neutrophil count, $\times 10 \square / \mathrm{L}$ & $4.5(3.1-6.6)$ & $3.7(2.6-5.2)$ & $6.1(3.8-8.7)$ & $<0.001$ \\
\hline$<1.8$ & $7 / 163(4.3 \%)$ & $5 / 76(6.6 \%)$ & $2 / 87(2.3 \%)$ & 0.170 \\
\hline $1.8-6.3$ & $111 / 163(68.1 \%)$ & $66 / 76(86.8 \%)$ & $45 / 87(51.7 \%)$ & .. \\
\hline$\geq 6.3$ & $45 / 163(27.6 \%)$ & $5 / 76(6.6 \%)$ & $40 / 87(46.0 \%)$ & .. \\
\hline Lymphocyte count, $\times 10 \square / \mathrm{L}$ & $0.9(0.6-1.3)$ & $1.3(0.9-1.6)$ & $0.7(0.5-1.0)$ & $<0.001$ \\
\hline$<0.8$ & $68 / 163(41.7 \%)$ & $16 / 76(21.1 \%)$ & $52 / 87(59.8 \%)$ & $<0.001$ \\
\hline Monocyte count, $\times 10 \square / \mathrm{L}$ & $0.50(0.32-0.66)$ & $0.51(0.37-0.69)$ & $0.46(0.28-0.62)$ & 0.061 \\
\hline Eosinophil count, $\times 10 \square / \mathrm{L}$ & $0.20(0.00-0.07)$ & $0.06(0.02-0.1)$ & $0.00(0.00-0.03)$ & $<0.001$ \\
\hline$<0.02$ & $72 / 163(44.2 \%)$ & $12 / 76(15.8 \%)$ & $60 / 87(69.0 \%)$ & $<0.001$ \\
\hline Hemoglobin, $\mathrm{g} / \mathrm{dL}$ & $127.0(117.0-137.0)$ & $124.0(115.0-132.8)$ & $131.0(118.0-142.0)$ & 0.009 \\
\hline Platelet count, $\times 10 \square / \mathrm{L}$ & $238.5(174.8 .0-305.3)$ & $285.0(226.0-355.0)$ & $202.0(202.0-280.0)$ & $<0.001$ \\
\hline$<100$ & $5 / 163(3.1 \%)$ & $2 / 76(2.6 \%)$ & 3/87 (3.4\%) & 0.564 \\
\hline Alanine transaminase, $\mathrm{U} / \mathrm{L}$ & $26.0(18.0-46.0)$ & $24.0(16.3-42.5)$ & $28.0(21.0-48.0)$ & 0.078 \\
\hline Aspartate transaminase, $\mathrm{U} / \mathrm{L}$ & $30.0(21.0-48.0)$ & $26.0(19.0-37.0)$ & $35.0(24.0-51.0)$ & 0.001 \\
\hline$>40$ & $50 / 163(30.7 \%)$ & 15/76 (19.7\%) & $35 / 87(40.2 \%)$ & 0.004 \\
\hline Albumin, $g / L$ & $33.4(30.4-36.1)$ & $34.4(31.4-37.3)$ & $32.5(30.0-35.1)$ & 0.004 \\
\hline$<32$ & $61 / 163(37.4 \%)$ & $21 / 76(27.6 \%)$ & $40 / 87(46.0 \%)$ & 0.012 \\
\hline Prealbumin, mg/L & $116.0(80.0-179.0)$ & $176.5(86.0-212.0)$ & $98.0(80.0-160.0)$ & 0.026 \\
\hline Total bilirubin, $\mu \mathrm{mol} / \mathrm{L}$ & $9.6(7.2-13.0)$ & $8.4(6.3-11.3)$ & $11.0(8.1-14.7)$ & $<0.001$ \\
\hline Direct bilirubin, $\mu \mathrm{mol} / \mathrm{L}$ & $4.4(3.1-6.1)$ & $3.7(2.7-5.0)$ & $5.4(3.7-6.9)$ & $<0.001$ \\
\hline Indirect bilirubin, $\mu \mathrm{mol} / \mathrm{L}$ & $4.9(3.6-7.1)$ & $4.5(3.3-6.4)$ & $5.4(4.1-7.9)$ & 0.009 \\
\hline Lactate dehydrogenase, $\mathrm{U} / \mathrm{L}$ & $314.0(262.6-426.3)$ & $258.0(223.5-292.5)$ & $393.0(326.0-506.5)$ & $<0.001$ \\
\hline$>225$ & $137 / 160(85.6 \%)$ & $56 / 76(73.7 \%)$ & $81 / 84(96.4 \%)$ & $<0.001$ \\
\hline ४-glutamyl transpeptidase, U/L & $32.0(21.0-69.0)$ & $32.0(19.0-60.0)$ & $37.5(21.0-78.0)$ & 0.231 \\
\hline Blood urea nitrogen, $\mathrm{mmol} / \mathrm{L}$ & $5.1(3.8-6.5)$ & $4.6(3.2-6.0)$ & $5.1(4.1-7.0)$ & 0.729 \\
\hline Creatinine, $\mu \mathrm{mol} / \mathrm{L}$ & $73.0(59.0-86.0)$ & $69.0(57.0-79.3)$ & $78.0(62.0-92.0)$ & $<0.001$ \\
\hline$>104$ & $16 / 161(9.9 \%)$ & $3 / 74(4.1 \%)$ & $13 / 87(16.0 \%)$ & 0.018 \\
\hline Potassium, mmol/L & $4.22(3.79-4.53)$ & $4.22(3.72-4.72)$ & $4.18(3.80-4.48)$ & $<0.001$ \\
\hline Sodium, $\mathrm{mmol} / \mathrm{L}$ & $138.6(135.9-141.0)$ & $139.5(137.6-141.6)$ & $137.8(134.4-139.6)$ & 0.357 \\
\hline Calcium, mmol/L & $2.11(2.05-2.18)$ & $2.15(2.06-2.20)$ & $2.09(2.04-2.16)$ & $<0.001$ \\
\hline Blood glucose, mmol/L & $6.1(5.1-8.1)$ & $5.4(4.7-6.3)$ & $6.9(5.8-8.9)$ & $<0.001$ \\
\hline$>7.00$ & $55 / 152(36.2 \%)$ & $15 / 71(21.2 \%)$ & $40 / 81(49.4 \%)$ & $<0.001$ \\
\hline Prothrombin time, seconds & $13.9(13.6-14.6)$ & $13.7(13.4-14.1)$ & $14.2(13.7-14.9)$ & $<0.001$ \\
\hline
\end{tabular}




\begin{tabular}{|c|c|c|c|c|}
\hline Activated partial thromboplastin time, seconds & $38.4(35.2-42.4)$ & $38.3(35.6-42.2)$ & $39.0(35.0-43.0)$ & 0.434 \\
\hline International normalized ratio & $1.07(1.02-1.14)$ & $1.05(1.01-1.09)$ & $1.09(1.04-1.15)$ & 0.001 \\
\hline D-dimer, $\mu \mathrm{g} / \mathrm{mL}$ & $1.27(0.66-2.68)$ & $0.95(0.47-1.90)$ & $1.72(0.77-6.27)$ & $<0.001$ \\
\hline$<0.5$ & $26 / 153(17.0 \%)$ & $20 / 75$ (26.7\%) & $6 / 76(7.9 \%)$ & 0.002 \\
\hline $0.5-21$ & $119 / 153(77.8 \%)$ & $54 / 75$ (72.0\%) & $65 / 78(83.3 \%)$ & .. \\
\hline$\geq 21$ & $8 / 153(5.2 \%)$ & $1 / 75(1.3 \%)$ & $7 / 83(8.4 \%)$ & .. \\
\hline High-sensitivity cardiac troponin I, ng/L & $6.1(2.90-12.9)$ & $3.6(1.9-8.3)$ & $7.5(4.2-19.9)$ & $<0.001$ \\
\hline$\geq 34.2$ & $9 / 119(7.6 \%)$ & $1 / 52(1.9 \%)$ & $8 / 67$ (11.9\%) & 0.080 \\
\hline Myoglobin, ng/mL & $56.5(28.8-91.1)$ & $37.3(24.5-59.4)$ & $80.9(49.7-129.3)$ & $<0.001$ \\
\hline Creatine kinase isoenzyme-MB, ng/ML & $0.7(0.5-1.5)$ & $0.6(0.3-1.1)$ & $0.9(0.6-1.9)$ & 0.008 \\
\hline $\mathrm{N}$-terminal pro-B-type natriuretic peptide, $\mathrm{pg} / \mathrm{m}$ & $192.0(70.6-442.0)$ & $76.5(45.8-270.8)$ & $298.0(162.5 .8-777.5)$ & $<0.001$ \\
\hline$>161$ & $65 / 114(57.0 \%)$ & $19 / 52$ (36.5\%) & $46 / 62(74.2 \%)$ & $<0.001$ \\
\hline High sensitivity C-reactive protein, $\mathrm{pg} / \mathrm{mL}$ & $39.3(11.2-86.3)$ & $12.4(4.2-40.3)$ & $63.5(33.6-127.7)$ & $<0.001$ \\
\hline$>10$ & $97 / 127(76.4 \%)$ & $32 / 57(56.1 \%)$ & $65 / 70(92.9 \%)$ & $<0.001$ \\
\hline Erythrocyte sedimentation rate, $\mathrm{mm} / \mathrm{h}$ & $47.0(27.0-71.0)$ & $53.0(32.5-77.8)$ & $46.0(25.0-68.0)$ & 0.435 \\
\hline Procalcitonin, ng/mL & $0.08(0.04-0.13)$ & $0.05(0.03-0.10)$ & $0.09(0.06-0.22)$ & $<0.001$ \\
\hline$<0.05$ & $30 / 113(26.5 \%)$ & $22 / 48(45.8 \%)$ & $8 / 65(12.3 \%)$ & 0.273 \\
\hline $0.05-0.5$ & $74 / 113(65.5 \%)$ & $25 / 48(52.1 \%)$ & $49 / 65(75.4 \%)$ &.. \\
\hline $0.5-2$ & $8 / 113(7.1 \%)$ & $1 / 48(2.1 \%)$ & $7 / 65(10.8 \%)$ &.. \\
\hline$\geq 2$ & $1 / 113(0.0 \%)$ & $0 / 48(0.0 \%)$ & $1 / 65(1.5 \%)$ & .. \\
\hline Serum ferritin, $\mu \mathrm{g} / \mathrm{L}$ & $841.6(526.2-1591.5)$ & $476.0(248.9-788.1)$ & $1245.0(757.4-1832.5)$ & 0.026 \\
\hline$\geq 400$ & $38 / 45(84.4 \%)$ & $9 / 16(56.3 \%)$ & $29 / 29$ (100.0\%) & $<0.001$ \\
\hline Interleukin-1 $\beta, \mathrm{pg} / \mathrm{mL}$ & $5.0(5.0-5.0)$ & $5.0(5.0-5.0)$ & $5.0(5.0-5.0)$ & 0.198 \\
\hline$\geq 5 \mathrm{pg} / \mathrm{mL}$ & $7 / 67(10.5 \%)$ & $1 / 29(3.4 \%)$ & 6/38 (15.8\%) & 0.129 \\
\hline Interleukin-2 receptor, U/L & $690.5(496.6-981.8)$ & $556.0(491.5-727.0)$ & $838.0(566.5-1059.0)$ & 0.011 \\
\hline$\geq 710$ & $30 / 66(45.5 \%)$ & $7 / 29(24.1 \%)$ & $23 / 37(62.2 \%)$ & 0.002 \\
\hline Interleukin-6, pg/mL & $15.7(3.4-48.8)$ & $5.3(2.4-16.9)$ & $37.1(6.2-81.6)$ & 0.001 \\
\hline$\geq 7$ & $38 / 67(56.7 \%)$ & $11 / 30(36.7 \%)$ & $27 / 37(73.0 \%)$ & 0.003 \\
\hline Interleukin-8, $\mathrm{pg} / \mathrm{mL}$ & $14.4(8.0-28.5)$ & $12.1(5.3-19.2)$ & $19.0(9.3-40.1)$ & 0.019 \\
\hline$\geq 62 \mathrm{pg} / \mathrm{mL}$ & 7/66 (10.6\%) & $2 / 29(6.9 \%)$ & $5 / 37(13.5 \%)$ & 0.453 \\
\hline Interleukin-10, pg/mL & $5.1(5.0-9.5)$ & $5.0(5.0-6.8)$ & $7.3(5.0-11.2)$ & 0.044 \\
\hline$\geq 9.2 \mathrm{pg} / \mathrm{mL}$ & $17 / 66(25.8 \%)$ & $4 / 29(13.8 \%)$ & $13 / 37(35.1 \%)$ & 0.087 \\
\hline Tumour necrosis factor $\alpha, \mathrm{pg} / \mathrm{mL}$ & $8.5(6.6-11.0)$ & $7.5(5.7-10.6)$ & $9.3(7.5-11.2)$ & 0.026 \\
\hline$>8.1$ & $35 / 64(54.7 \%)$ & $10 / 28(35.7 \%)$ & $25 / 36(69.4 \%)$ & 0.007 \\
\hline
\end{tabular}

Abbreviations: COVID-19 coronavirus disease 2019. Data were median (IQR) or n/N (\%), where $\mathrm{N}$ was the total number of patients with available data. $P$ values comparing moderate and severe cases were from $\chi \llbracket$ test, Fisher's exact test, or Mann-Whitney U test. $P<0.05$ was considered statistically significant. 
Table 3 Treatments of moderate and severe patients with COVID-19

\begin{tabular}{|c|c|c|c|c|}
\hline Treatment & All patients $(n=163)$ & Moderate cases $(n=76)$ & Severe cases $(n=87)$ & $P$ \\
\hline Antiviral therapy & 155/163 (95.1\%) & $73 / 76(96.0 \%)$ & $82 / 87(94.2 \%)$ & 0.725 \\
\hline Antibiotics & $142 / 163(87.1 \%)$ & $57 / 76(76.3 \%)$ & 85/87 (97.7\%) & $<0.001$ \\
\hline Glucocorticoid & 71/163 (43.6\%) & $6 / 76(7.9 \%)$ & $65 / 87$ (74.7\%) & $<0.001$ \\
\hline Intravenous albumin & $27 / 163(16.6 \%)$ & $6 / 76(7.9 \%)$ & $21 / 87$ (24.1\%) & 0.005 \\
\hline Intravenous immunoglobulin & $44 / 163(27.0 \%)$ & $5 / 76(6.6 \%)$ & $39 / 87$ (44.8\%) & $<0.001$ \\
\hline Thymosin & $14 / 163(8.6 \%)$ & $2 / 76(2.6 \%)$ & $12 / 87$ (13.8\%) & 0.011 \\
\hline Low molecular weight heparin & $20 / 163(12.3 \%)$ & $2 / 76(2.6 \%)$ & $18 / 87(20.1 \%)$ & $<0.001$ \\
\hline \multicolumn{5}{|l|}{ Oxygen treatment } \\
\hline High flow nasal cannula & $72 / 163(44.2 \%)$ & $0 / 76(0.0 \%)$ & $72 / 87$ (82.8\%) & $<0.001$ \\
\hline Non-invasive mechanical ventilation & $28 / 163(17.2 \%)$ & $0 / 76(0.0 \%)$ & $28 / 87(32.2 \%)$ & $<0.001$ \\
\hline
\end{tabular}

Abbreviations: COVID-19 coronavirus disease 2019. Data were $\mathrm{n} / \mathrm{N}(\%)$, where $\mathrm{N}$ was the total number of patients with available data. $P$ values comparing moderate and severe cases were from $\chi \square$ test or Fisher's exact test. $P<0.05$ was considered stratistically significant.

Table 4 Univariate and multivariable analysis of factors for discrimination of disease severity

\begin{tabular}{lccccccc}
\hline \multirow{2}{*}{ Factors } & \multicolumn{5}{c}{ Univariable } & \multicolumn{3}{c}{ Multivariable } \\
\cline { 2 - 7 } & Odd ratio & $95 \%$ CI & $P$ value & Odd ratio & $95 \%$ CI & $P$ \\
\hline Sex (male) & 1.99 & $1.07,3.72$ & 0.031 &.. &.. &.. \\
\hline Hypertension & 2.32 & $1.22,4.41$ & 0.01 &.. &.. &.. \\
\hline Neutrophil count & 1.48 & $1.26,1.74<0.001$ & 1.34 & $1.07,1.69$ & 0.012 \\
\hline Eosinophil count $(<0.04 \times 10 \square / \mathrm{L})$ & 10.4 & $4.84,21.74<0.001$ & 19.93 & $5.03,50.41<0.001$ \\
\hline Lymphocyte count & 0.034 & $0.01,0.12<0.001$ &.. &.. &.. \\
\hline Lactate dehydrogenase & 1.02 & $1.01,1.02<0.001$ & 1.02 & $1.01,1.02<0.001$ \\
\hline Albumin & 0.9 & $0.83,0.97$ & 0.006 &.. &.. &.. \\
\hline Creatinine & 1.02 & $1.00,1.03$ & 0.021 &.. &.. &.. \\
\hline Prothrombin time & 2.48 & $1.59,3.88<0.001$ &.. &.. &.. \\
\hline D-dimer & 1.19 & $1.07,1.33$ & 0.002 & 1.15 & $1.01,1.32$ & 0.038 \\
\hline
\end{tabular}

Abbreviations: COVID-19 coronavirus disease 2019 
Table 5 ROC curve results of factors for discrimination of disease severity

\begin{tabular}{|c|c|c|c|c|c|}
\hline Factors & Cut-off value & Sensitivity & Specificity & AUC & AUC 95\%CI \\
\hline Neutrophil count, $\times 10 \square / L$ & 5.97 & $51.7 \%$ & $88.2 \%$ & 0.74 & $0.67,0.81$ \\
\hline Eosinophil count, $\times 10 \square / \mathrm{L}$ & 0.04 & $82.8 \%$ & $68.4 \%$ & 0.76 & $0.68,0.83$ \\
\hline $\mathrm{LDH}, \mathrm{U} / \mathrm{L}$ & 310 & $83.3 \%$ & $84.2 \%$ & 0.86 & $0.80,0.92$ \\
\hline D-dimer, $\mu \mathrm{g} / \mathrm{mL}$ & 3.34 & $33.3 \%$ & $97.3 \%$ & 0.69 & $0.61,0.77$ \\
\hline Neutrophil + Eosinophil + LDH + D-dimer & .. & $88.0 \%$ & $81.3 \%$ & 0.93 & $0.89,0.97$ \\
\hline
\end{tabular}

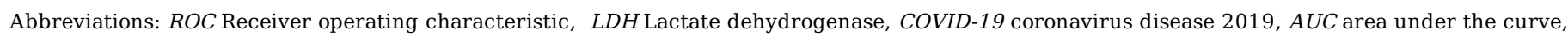

Figures
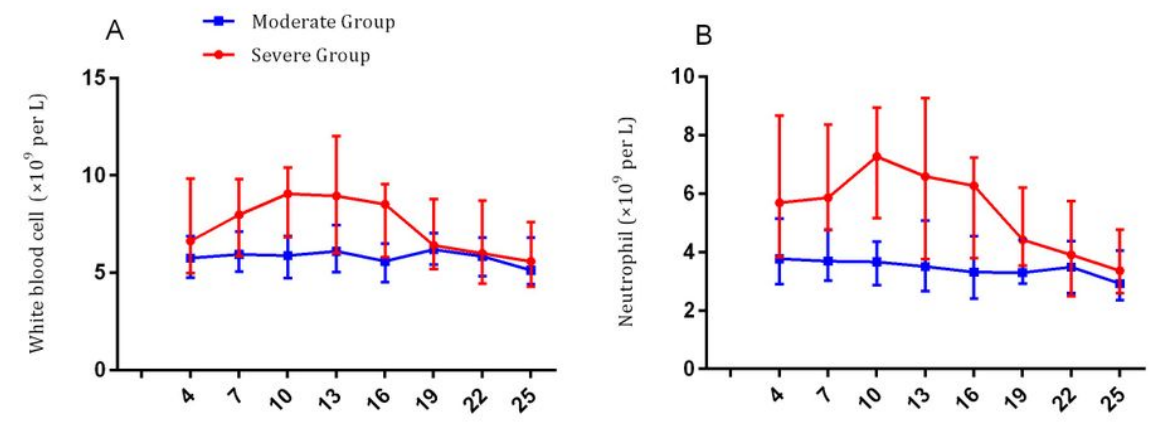

C

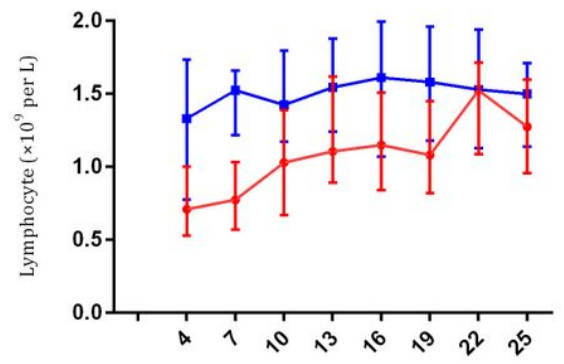

D
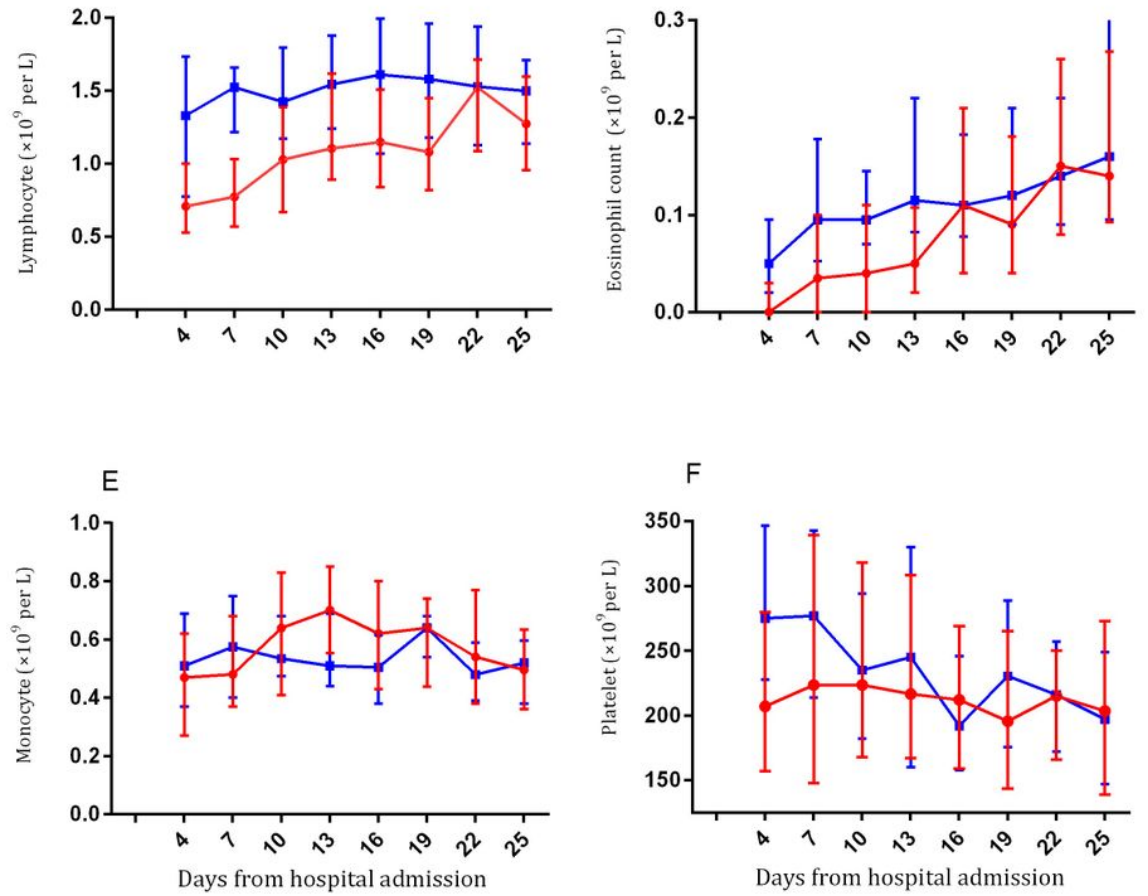

Figure 1 
Temporal changes of blood cell counts during hosiptalization in patients with COVID-19. Figure showed the temporal changes in WBC (A), neutrophils (B), lymphocytes (C), monocytes (D), eosinophils (E) and platelets (F) of moderate and severe cases from hospital admission to discharge. Differences in WBC, neutrophils, lymphocytes and eosinophils between two groups were significant from admission to day 19 after admission, except for day 16 for eosinophils and day 19 for WBC and eosinophils. COVID-19 coronavirus disease 2019, WBC white blood cells.

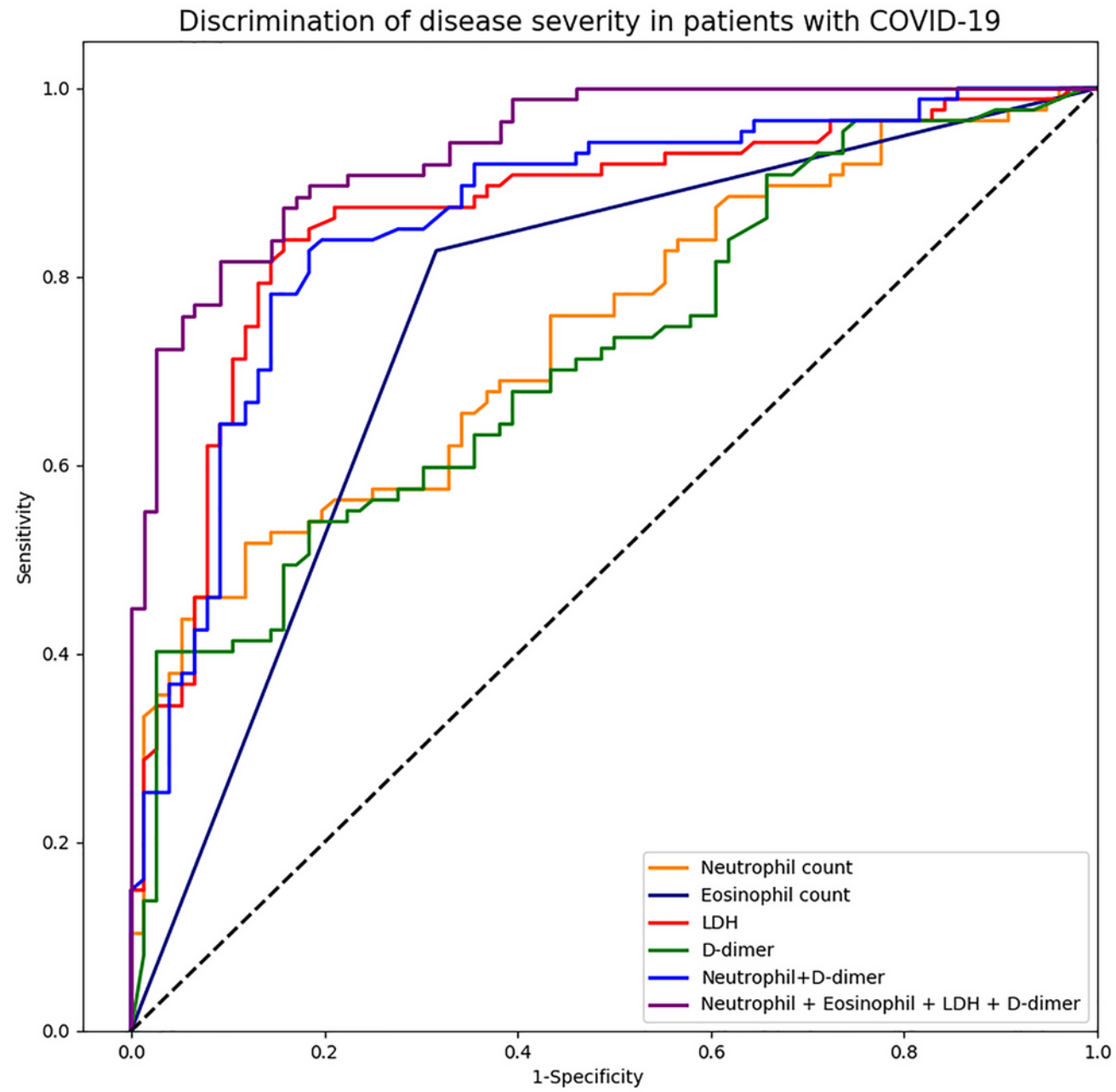

Figure 2

ROC curves of neutrophil count, eosinophil (< $0.04 \times 109 / L)$, LDH, D-dimer, and neutrophil + eosinophil + LDH + D-dimer combination on admission for discrimination of moderate and severe cases of COVID-19. ROC receiver operating characteristic, LDH lactate dehydrogenase, COVID-19 coronavirus disease 2019.

\section{Supplementary Files}

This is a list of supplementary files associated with this preprint. Click to download.

- SupplementaryTable1.pdf 